\title{
Relações entre as características morfométricas da carcaça e a produção de cortes comerciais de novilhas da raça Nelore
}

\author{
Sanches, L.M. ${ }^{\circledR} ;$ Fernandes, A.R.M. e Borquis, R.R.A.
}

Departamento de Produção Animal. Universidade Federal da Grande Dourados. Dourados. Brasil.

\section{PALAVRASCHAVEADICIONAIS}

Correlação.

Fêmeas.

Medidas de carcaca.

Predição.

Zebuínos.

\section{ADDITIONAL KEYWORDS}

Carcass measurements.

Correlation.

Female.

Predicition.

Zebu.

\section{INFORMATION}

Cronología del artículo.

Recibido/Received: 26.06.2019

Aceptado/Accepted: 23.03 .2021

On-line: 15.04 .2021

Correspondencia a los autores/Contact e-mail:

lorenamarisanches@hotmail.com.

\section{RESUMO}

Um estudo de correlações foi conduzido para determinar quais as principais medidas morfométricas da carcaça que influenciam na determinação dos cortes comerciais do dianteiro e traseiro especial em novilhas nelore. Utilizou-se 81 novilhas nelore, escolhidas aleatoriamente provenientes de rebanhos comerciais, abatidas de acordo com a legislação vigente. Foram coletados os pesos corporais dos animais e posteriormente ao abate coletou-se o peso da carcaça quente, peso da carcaça fria e as mesmas foram divididas em meias carcaças e pesadas após o resfriamento. As medidas morfométricas da carcaça foram comprimento interno da carcaça, comprimento, largura, perímetro e profundidade de perna, profundidade de peito, área de olho de lombo e espessura de gordura subcutânea. As meias carcaças foram dividas em dianteiro e traseiro especial com intuito de estabelecer os cortes comerciais. Os dados foram submetidos à análise de correlação de Pearson $(p<0,05)$ e posteriormente desenvolvidos equações de regressão linear múltipla pelo método stepwise. As maiores correlações positivas $(<0,60)$ foram referentes à variável peso, sendo que a melhor correlação dos cortes do dianteiro e traseiro especial foi com o peso da meia carcaça, as variáveis referentes ao comprimento, largura, profundidade e perímetro apresentaram correlações baixas às moderadas $(<0,40)$, a área de olho de lombo apresentou correlação moderada e positiva $(<0,40)$, e a espessura de gordura subcutânea não apresentou correlação com os cortes comerciais. O peso da meia carcaça foi a primeira variável que participou do modelo de regressão linear múltipla para o dianteiro e traseiro especial, as demais variáveis estudadas não apresentaram um acréscimo alto no modelo de predição. Os valores observados e preditos foram calculados e comparados, os mesmos foram amplamente semelhantes mostrando assim a alta predição das equações de regressão, podendo ser bem aplicadas para estimar os cortes do dianteiro e traseiro especial. A variável mais importante para os modelos de predição dos cortes comerciais de novilhas nelore foi o peso da meia carcaça.

\section{Relationship between morphometric carcass characteristics and the production of commercial meat cuts of Nellore heifers}

\section{SUMMARY}

A study of correlations was conducted to determine the main morphometric measurements of the carcass that influence the determination of the commercial cuts from the forequarter and hindquarter in Nellore heifers. Eighty-one Nellore heifers, randomly chosen form a commercial herd, were slaughtered according to the actual regulation. The animal's body weight was measured before the slaughter. After the slaughte ring, the hot and cold carcasses were determined and then divided into half-carcasses and weighed after cooling. The morphometric measurements evaluated were the internal carcass length, width, perimeter and depth of leg, chest depth, ribeye area, and subcutaneous fat thickness. The half-carcasses were divided into forequarter and hindquarter to establish the commercial meat cuts. The data were analysed using Pearson's correlation $(p<0,05)$, followed by the development of multiple linear equations using the stepwise method. The highest positive correlations $(>0.60)$ were found in the variable weight, as the best correlation between the cuts of the forequarter and hindquarter was with the half carcass weight. The variables related to length, width, depth, and perimeter presented low to moderate correlations $(<0.40)$ as the ribeye area showed a moderate and positive correlation $(<0.40)$, yet the subcutaneous fat thickness did not correlate with the commercial meat cuts. The weight of the half carcass was the first variable that participated in the multiple linear regression model for the forequarter and hindquarte, and the other tested variables did not show a high increase in the prediction model. The observed and predicted values were calculated and compared, and they were broadly similar, thus showing the high prediction of the regression equations, and it can be well applied to estimate the cuts of the forequarter and hindquarter. The most important variable to predict the commercial cuts of Nellore heifers was the half-carcass weight. 


\section{INTRODUÇÃO}

A pecuária bovina é uma das atividades com maior relevância para os setores do agronegócio brasileiro e da economia nacional. O Brasil possui alto potencial de produção no setor pecuário, por apresentar o maior rebanho comercial de bovinos, ocupando a posição de maior país exportador e o segundo maior produtor de carne bovina no mundo (Ribeiro et al., 2015). Expondo a necessidade de produzir cada vez mais a fim de elevar a produtividade e aperfeiçoar o processo de comercialização.

Dentre os principais problemas da indústria da carne bovina no Brasil estão à falta de uniformização dos animais, cobertura de gordura e marmorização da carne, no qual são fatores que exercem grandes influências na qualidade da carne (Lage et al., 2012). Sendo assim, a fim de atender os mercados internos e externos, existe a necessidade de se produzir animais com uma boa qualidade de carcaça, que apresentam maior rendimento de cortes comerciais e uma boa cobertura de gordura.

Desta forma, grande parte dos produtores brasileiros assumiram estratégias de produção de novilhas, pois atingem o peso de abate em um período parcialmente curto, ou seja, entre 20-24 meses de idade (Lage et al., 2012). Paulino et al. (2008), explicaram que o efeito da classe sexual é altamente determinante de diferenças no crescimento e nas taxas dos diferentes tecidos corporais e da carcaça, uma vez que machos inteiros apresentam maior taxa de crescimento (10 a 20\%) em relação a machos castrados e fêmeas que possuem carcaças acabadas em um menor período de tempo, devido a precocidade de deposição de gordura dos mesmos, sendo necessário estudos sobre características de carcaça para a classe sexual.

Entretanto, para melhorar o processo de comercialização e agregar valor ao produto é indispensável estabelecer um sistema de avaliação que auxilie na determinação do valor das carcaças produzidas em função da produção de carne, sendo necessário definir os parâmetros que melhor avaliem as diferenças entre o sexo dos animais. Logo as medidas realizadas na carcaça são importantes por si só, permitindo comparações entre os tipos raciais, pesos, sexo e idades ao abate, e pelas suas correlações com outras medidas como os tecidos constituintes da carcaça, possibilitando estimar suas características (Pinheiro \& Jorge, 2010).

Portanto, as medidas morfométricas da carcaça (comprimento, largura, perímetro e profundidade), possui correlação com os cortes comerciais em animais machos, a partir disso a hipótese do presente estudo foi avaliar a eficácia dessas correlações em fêmeas bovinas. O objetivo do presente estudo foi avaliar as correlações existentes entre as medidas morfométricas das carcaças e produção de cortes comerciais, bem como desenvolver modelos de predição da produção de cortes a partir da morfologia da carcaça.

\section{MATERIAL E MÉTODOS}

\section{COLETA DE DADOS}

Foram utilizadas 81 novilhas da raça Nelore com peso médio de $364 \mathrm{~kg}$, provenientes de rebanhos comerciais, terminadas em pastagem com a oferta de suplementação alimentar e mineral. Os animais passaram por um período de jejum sólido de 14 horas e foram escolhidos aleatoriamente na chegada ao frigorifico comercial Frigoverma, localizado no município de Ivinhema- Mato Grosso do Sul.

Após a chegada dos animais no frigorifico, os mesmos foram pesados através de uma balança mecânica, que possuía capacidade para 1500 kg e posteriormente abatidos, de acordo as normas do Regulamento da Inspeção Industrial e Sanitária de Produtos de Origem Animal - RIISPOA (BRASIL,1980), pelo método de atordoamento por concussão cerebral, com o auxílio de uma pistola de ar comprimido. Em seguida foi realizado o processo de sangria por secção jugular. No momento do abate foram coletadas as seguintes variáveis para todos os animais; peso de carcaça quente, gordura pélvica, perirrenal e inguinal, medidas de comprimento e profundidade da carcaça.

As carcaças foram divididas em meias-carcaças e encaminhadas para a câmara fria a $0^{\circ} \mathrm{C}$, por aproximadamente 24 horas. Ao decorrer este tempo as meias-carcaças passaram novamente pelo processo de pesagem a fim de obter o peso da carcaça resfriada.

As medidas morfométricas realizadas na carcaça foram: comprimento da carcaça (borda anterior do púbis até a borda anterior da primeira costela), profundidade do peito (maior distância entre o peito e o processo espinhoso do animal), comprimento da perna (cabeça do fêmur até a superfície articular meta tarsiana), largura da perna (ponto de maior largura do coxão do animal em sentido bíceps femural/ semimenbranoso), profundidade da perna (ponto de maior profundidade do coxão do animal no sentido quadríceps/semitendinoso), perímetro peito (maior circunferência obtida no peito do animal) e perímetro perna (maior circunferência obtida na perna do animal). A área do musculo Longissimus foi determinada utilizando-se uma régua de quadrantes de pontos e a espessura de gordura subcutânea através de um paquímetro (Suguisawa et al., 2006).

As meias-carcaças foram divididas em traseiro e dianteiro (Rezende et al., 2013). A separação do dianteiro foi realizada por meio de uma incisão entre a quinta e sexta costela, atingindo a região esternal (peito) e a coluna vertebral, na altura do quinto espaço intervertebral. A ponta de agulha foi removida a partir do quarto traseiro, através e uma distancia de $20 \mathrm{~cm}$ da coluna vertebral, resultando em outro quarto denominado traseiro especial, no qual é bem evidenciado por massas musculares que recobrem as últimas oito costelas, a última esternébra, o apêndice xifoide e a região do vazio. 
Os cortes primários provenientes do quarto dianteiro e traseiro foram subdivididos de acordo com os cortes comerciais estabelecidos pela legislação brasileira: filé mignon; picanha; coração da alcatra; maminha; contrafilé; coxão mole; coxão duro; lagarto; patinho; acém; pescoço; ponta de peito; miolo da paleta; peixinho; cupim e costelas.

Na Tabela I está apresentada a tabela descritiva das variáveis analisadas no presente trabalho (medidas da carcaça e cortes comerciais).

\section{ANÁLISE ESTATÍSTICA}

A análise estatística foi conduzida utilizando-se o pacote estatístico $R$ versão 3.3.1. As correlações foram feitas por meio de análise de correlações de Pearson, utilizando as medidas morfométricas da carcaça (variáveis independentes) e cortes comerciais do dianteiro e traseiro especial (variáveis dependentes), sendo consideradas significativas quando $\mathrm{p}<0,05$ de modo que $\mathrm{P}$ é o nível de significância associado à estatística calculada. As correlações fo-

Tabela I. Análise descritiva das variáveis associadas com as medidas da carcaça. (Descriptive analysis of the variables associated with carcass measurements).

\begin{tabular}{|c|c|c|c|c|c|}
\hline Variáveis & Média & $\mathrm{DP}$ & Mínimo & Máximo & C.V $(\%)$ \\
\hline $\mathrm{PC}, \mathrm{kg}$ & 363,14 & 34,60 & 293,00 & 475,32 & 9,53 \\
\hline $\mathrm{AOL}, \mathrm{cm}^{2}$ & 46,37 & 8,27 & 28,90 & 65,23 & 17,84 \\
\hline EGS, mm & 6,20 & 1,29 & 3,10 & 9,30 & 20,80 \\
\hline$P C Q, \mathrm{~kg}$ & 202,10 & 19,20 & 167,79 & 243,00 & 9,50 \\
\hline PCF, kg & 196,38 & 18,67 & 162,60 & 293,00 & 9,51 \\
\hline $\mathrm{RCQ}, \mathrm{kg}$ & 50,39 & 6,46 & 40,33 & 60,48 & 12,82 \\
\hline \multicolumn{6}{|l|}{ MEIA CARCAÇA } \\
\hline PMC, kg & 98,16 & 9,13 & 81,30 & 119,50 & 9,30 \\
\hline C.Int, cm & 127,53 & 4,58 & 118,00 & 135,00 & 3,59 \\
\hline C.Per, cm & 83,15 & 3,42 & 77,00 & 90,00 & 4,11 \\
\hline L.Per, cm & 25,29 & 2,40 & 20,00 & 30,00 & 9,48 \\
\hline PE.Per, cm & 88,31 & 9,08 & 71,50 & 120,00 & 10,29 \\
\hline P.Per, cm & 29,39 & 3,45 & 22,00 & 37,00 & 11,75 \\
\hline P.Pei, cm & 67,44 & 5,01 & 60,00 & 89,00 & 7,44 \\
\hline \multicolumn{6}{|c|}{ CORTES COMERCIAIS } \\
\hline Pescoço, kg & 6,22 & 0,80 & 4,80 & 8,60 & 12,80 \\
\hline Acém, kg & 5,55 & 0,84 & 3,55 & 8,15 & 15,16 \\
\hline M.P, kg & 4,44 & 0,58 & 3,30 & 6,00 & 13,06 \\
\hline M.D, kg & 3,44 & 0,38 & 2,80 & 4,60 & 11,06 \\
\hline C.P, kg & 0,65 & 0,19 & 0,30 & 1,20 & 29,93 \\
\hline Paleta & 3,09 & 0,37 & 2,10 & 3,80 & 12,15 \\
\hline CD/Lagarto, kg & 6,11 & 0,64 & 4,60 & 7,50 & 10,53 \\
\hline Maminha, kg & 1,16 & 0,19 & 0,80 & 1,60 & 16,83 \\
\hline Alcatra, kg & 3,13 & 0,32 & 2,40 & 3,80 & 10,37 \\
\hline Picanha, kg & 1,52 & 0,27 & 0,40 & 2,10 & 17,85 \\
\hline Patinho, kg & 4,47 & 0,47 & 3,40 & 5,70 & 10,41 \\
\hline Coxão Mole, kg & 7,50 & 0,78 & 6,00 & 9,50 & 10,41 \\
\hline Contra Filé, kg & 6,82 & 0,85 & 4,80 & 9,10 & 12,44 \\
\hline Filé Mignon, kg & 1,50 & 0,29 & 1,05 & 2,80 & 19,23 \\
\hline M.T, kg & 3,30 & 0,33 & 2,55 & 4,05 & 9,88 \\
\hline
\end{tabular}

$\mathrm{DP}=$ desvio padrão; $\mathrm{C} . \mathrm{V}(\%)=$ coeficiente de variação; $\mathrm{PC}=$ peso corporal; $\mathrm{AOL}=$ área do músculo Longissimus; $E G S=$ espessura de gordura subcutânea; $P C Q=$ peso da carcaça quente; $P C F=$ peso da carcaça fria; $R C Q=$ rendimento de carcaça quente; $P M C=$ peso da meia carcaça; C. Int= comprimento interno; C.Per= comprimento da perna; L.Per= largura da perna; PE.Per= perímetro perna; P.Per=Profundidade perna; P.Pei= profundidade peito; $M . P=$ miolo da paleta; $M . D=$ músculo do dianteiro; $C . P=$ capa da paleta; $C D / L a g a r t o=$ corte do coxão duro; M.T=músculo traseiro. 
ram consideradas baixas quando apresentaram um valor $<0,40$, moderadas quando apresentaram um valor entre $0,41-0,59$ e a altas quando apresentaram um valor $>0,60$. De acordo com o seguinte modelo matemático de correlação de Pearson:

$$
\mathrm{r}(\mathrm{x}, \mathrm{y})=
$$

$\mathrm{X}=$ variáveis independentes (medidas morfométricas da carcaça);

$\mathrm{Y}=$ variáveis dependentes (cortes comerciais);

$\mathrm{N}=$ número total de variáveis;

As equações de predição para os cortes comerciais do dianteiro e traseiro especial (variáveis dependentes) foram desenvolvidas em um modelo de regressão linear múltipla, utilizando o procedimento stepwise, que seleciona em um primeiro passo a variável independente que gera o melhor modelo, usando as medidas morfométricas da carcaça como variáveis independentes. A capacidade preditiva das equações desenvolvidas foi determinada pelo coeficiente de determinação $\left(\mathrm{R}^{2}\right)$. Os valores observados foram calculados a partir dos dados coletados e os valores preditos por meio das equações de regressão. Conforme o seguinte modelo matemático de regressão linear múltipla:

$$
\mathrm{Y}=\beta 0+\beta 1 \mathrm{X} 1+\ldots+\beta \mathrm{kXk}+\mathrm{E}
$$

$\mathrm{X} 1, \ldots, \mathrm{Xk}=$ variáveis independentes (medidas morfométricas da carcaça);

$\mathrm{E}=$ variável aleatória residual na qual se procuram incluir todas as influencias no comportamento da variável Y que não podem ser explicadas linearmente pelo comportamento das variáveis $\mathrm{X} 1, \ldots, \mathrm{Xk}$ e os possíveis erros de medição;

$$
\begin{aligned}
& \beta 0=\text { constante; } \\
& \beta 1 \ldots \beta \mathrm{k}=\text { coeficientes de regressão parciais; } \\
& \mathrm{Y}=\text { variável dependente (cortes comerciais); }
\end{aligned}
$$

\section{RESULTADOS}

O peso da meia carcaça apresentou correlações altas $(>0,60)$ com o peso dos cortes do dianteiro, seguida pelo peso da carcaça fria, carcaça quente e peso vivo do animal (Tabela II). Conforme observado no presente trabalho, existe uma alta correlação entre o peso corporal e de carcaça com os cortes comerciais, mostrando que essa variável pode ser utilizada para prever o peso dos cortes do dianteiro de fêmeas bovinas (Tabela II).

O comprimento interno da carcaça, largura da perna e área de olho de lombo exibiram correlações baixas $(<0,40)$ com os cortes comerciais do dianteiro. As demais medidas morfométricas da carcaça como, comprimento da perna, perímetro da perna, profundidade do peito e da perna e espessura de gordura subcutânea não se correlacionaram com os cortes do dianteiro (Tabela II), assim essas medidas não teriam influência direta para predizer a melhor equação referente aos cortes do dianteiro de novilhas Nelore.
Para o traseiro especial as melhores correlações $(>0,60)$ foram as referentes ao peso de carcaça, sendo que semelhante ao dianteiro à correlação mais alta $(>0,60)$ foi com o peso da meia carcaça $(0,93)$, seguida por carcaça fria $(0,90)$, carcaça quente $(0,88)$ e peso vivo $(0,82)$, ressaltando que o peso influência significativamente na retalhabilidade dos cortes comerciais do traseiro em fêmeas bovinas (Tabela III).

O comprimento interno da carcaça, comprimento da perna, largura da perna, profundidade do peito e área do musculo Longissimus apresentaram correlações moderadas $(<0,40)$ com os cortes do traseiro especial, exceto para o filé mignon (Tabela III). A profundidade da perna e a espessura de gordura subcutânea não apresentaram correlações significativas com os cortes do traseiro especial (Tabela III), mostrando independência entre essas medidas da carcaça e cortes cárneos comerciais do traseiro especial.

Na Tabela IV apresentam-se os modelos de predição para os cortes do dianteiro (CD) e cortes do traseiro especial (TE) utilizando as medidas obtidas na carcaça. O peso da meia carcaça foi a variável que obteve maior participação nos modelos de predição. As variáveis que contribuíram para a formação dos cortes do dianteiro foram o peso corporal, peso da meia carcaça e área de olho de lombo e para os cortes do traseiro especial foram o peso corporal, peso da meia carcaça, área de olho de lombo, comprimento da perna, perímetro da perna e espessura de gordura subcutânea. O coeficiente de determinação de ambos os quartos foram altos $84,13 \%$ para o dianteiro e $88,66 \%$ para o traseiro especial, ressaltando a eficácia das equações de regressão com a inclusão das medidas de carcaça para os modelos de predição.

Os modelos de predição para os cortes individuais do dianteiro estão apresentados na Tabela V. Para todos os cortes comerciais do dianteiro, o peso foi a primeira variável que contribuiu para os modelos de predição, comprovando as altas correlações, sendo assim se faz necessário à inclusão dessa variável para estimar a retalhabilidade dos cortes comerciais do dianteiro. A variável peso teve uma participação de $40,26 \%$ (PCQ) para o pescoço, $54,03 \%$ (PC) para o acém, 61,25\% (MC) para o miolo da paleta, 50,09\% (MC) para o músculo dianteiro e $58,86 \%$ (MC) para paleta.

As demais medidas realizadas na carcaça (comprimento, largura, profundidade, perímetro, área de olho de lombo e espessura de gordura subcutânea) participaram dos modelos de predição (Tabela V), porém essa contribuição não foi significativa comparada ao peso, ou seja, o peso foi a principal variável capaz de explicar maior parte das equações de regressão para formação dos cortes comerciais do dianteiro de novilhas.

Para os cortes comerciais do traseiro especial a primeira variável que contribuiu para a predição foi o peso, com 63,81\% (PCF) para a alcatra, 58,49\% (MC) para o contra filé 65,53\% (MC) para o lagarto, 77,68\% (MC) para o coxão mole, 39,09 (PCQ) para a maminha, 51,06\% (PV) para o músculo traseiro, 


\begin{tabular}{|c|c|c|c|c|c|c|c|c|c|c|c|c|c|}
\hline Variáveis & PC & $P C Q$ & PCF & $\mathrm{RCQ}$ & PMC & C.Int & C.Per & L.Per & P.Per & P.Pei & PE.Per & EGS & $\mathrm{AOL}$ \\
\hline Pescoço & $0,55^{\star \star \star}$ & $0,58^{* * *}$ & $0,64^{\star \star *}$ & $0,13^{\mathrm{NS}}$ & $0,64^{\star * *}$ & 0,23 & $0,28^{*}$ & $0,15^{\mathrm{NS}}$ & $-0,067^{\text {NS }}$ & $0,30^{*}$ & $0,08^{\mathrm{NS}}$ & 0,21 & $0,25^{*}$ \\
\hline Acém & $0,74^{* * *}$ & $0,71^{* * *}$ & $0,73^{\star \star *}$ & $-0,10^{\mathrm{NS}}$ & $0,73^{\star \star *}$ & $0,38^{\star *}$ & $0,27^{*}$ & $0,20^{\mathrm{NS}}$ & $0,13^{\mathrm{NS}}$ & $0,34^{\star *}$ & $0,081^{\mathrm{NS}}$ & $0,25^{\star}$ & $0,27^{*}$ \\
\hline C.P & 0,23 . & 0,23 & $0,27^{*}$ & $0,069^{\mathrm{NS}}$ & $0,28^{*}$ & $0,063^{\mathrm{NS}}$ & $0,15^{\mathrm{NS}}$ & $0,11^{\mathrm{NS}}$ & $-0,21$ & $0,34^{\star *}$ & $0,073^{\mathrm{NS}}$ & $0,081^{\text {NS }}$ & 0,22 \\
\hline M.P & $0,71^{\star \star *}$ & $0,77^{\star \star \star}$ & $0,77^{\star \star \star}$ & $0,28^{*}$ & $0,79^{* * *}$ & $0,33^{\star *}$ & $0,37^{\star \star}$ & $0,28^{*}$ & $0,12^{\mathrm{NS}}$ & 0,23 & $0,46^{\star \star *}$ & 0,24 & $0,24^{*}$ \\
\hline M.D & $0,59^{\star \star \star}$ & $0,69^{* \star *}$ & $0,66^{\star * *}$ & $0,012^{\mathrm{NS}}$ & $0,71^{\star * \star}$ & $0,29^{*}$ & $0,41^{* * *}$ & $0,14^{\mathrm{NS}}$ & $0,004^{\mathrm{NS}}$ & $0,37^{* *}$ & $0,30^{*}$ & $0,14^{\mathrm{NS}}$ & $0,29^{*}$ \\
\hline Paleta & $0,68^{* * *}$ & $0,73^{* \star \star}$ & $00,74^{\star \star \star}$ & $0,12^{\mathrm{NS}}$ & $0,77^{\star * \star}$ & $0,36^{\star *}$ & 0,23 & 0,23 & $0,05^{\mathrm{NS}}$ & $0,49^{* * *}$ & $0,25^{*}$ & 0,21 . & $0,38^{* *}$ \\
\hline C.D & $0,83^{\star \star *}$ & $0,86^{\star \star *}$ & $0,89^{* * *}$ & $0,098^{\mathrm{NS}}$ & $0,91^{* * *}$ & $0,28^{*}$ & $0,082^{\mathrm{NS}}$ & $0,26^{*}$ & $0,020^{\mathrm{NS}}$ & $-0,033^{\mathrm{NS}}$ & $-0,117^{\mathrm{NS}}$ & $-0,086^{\mathrm{NS}}$ & $0,35^{\star *}$ \\
\hline
\end{tabular}

$\mathrm{PC}=$ peso corporal; $\mathrm{PCQ}=$ peso da carcaça quente; $\mathrm{PCF}=$ peso da carcaça fria; $\mathrm{RCQ}=$ rendimento de carcaça quente; $\mathrm{PMC}=$ peso da meia carcaça; C. Int= comprimento interno; C.Per= comprimento da perna; L.Per= largura da perna; P.Per=Profundidade perna; P.Pei= profundidade peito; PE.Per= perímetro perna; $\mathrm{AOL}=$ área do músculo Longissimus; $E \mathrm{GS}=$ espessura de gordura subcutânea; $\mathrm{C} . \mathrm{P}=\mathrm{capa}$ da paleta; $M . P=$ miolo da paleta; $M . D=$ músculo do dianteiro; $C . D=$ cortes do dianteiro $\left({ }^{* *} P<0.001,{ }^{* *} P<0.01,{ }^{*} P<0.05, \cdot P<0.1\right)$.

Tabela III. Correlações entre as medidas de carcaça e os cortes comerciais do traseiro especial de novilhas Nelore. (Correlations between carcass measurements and commercial cuts of Nelore heifers).

\begin{tabular}{|c|c|c|c|c|c|c|c|c|c|c|c|c|c|}
\hline Variáveis & PC & $P C Q$ & PCF & $\mathrm{RCQ}$ & PMC & C.Int & C.Per & L.Per & P.Per & P.Pei & PE.Per & EGS & $\mathrm{AOL}$ \\
\hline Alcatra & $0,77^{\star \star \star}$ & $0,78^{* * *}$ & $0,80^{* * *}$ & $0,10^{\mathrm{NS}}$ & $0,83^{\star \star \star}$ & $0,37^{* *}$ & $0,42^{* * *}$ & 0,23 & $0,064^{\mathrm{NS}}$ & $0,47^{* * *}$ & 0,23 & $0,082^{\mathrm{NS}}$ & $0,26^{*}$ \\
\hline $\begin{array}{l}\text { Contra } \\
\text { Filé }\end{array}$ & $0,62^{\star \star *}$ & $0,76^{* * *}$ & $0,75^{\star * *}$ & $0,09^{N S}$ & $0,77^{\star \star *}$ & $0,36^{* *}$ & $0,27^{*}$ & $0,18^{\mathrm{NS}}$ & $0,17^{\mathrm{NS}}$ & $0,36^{* *}$ & $0,41^{* * *}$ & $0,067^{\mathrm{NS}}$ & $0,32^{* *}$ \\
\hline $\begin{array}{l}\text { CD/La- } \\
\text { garto }\end{array}$ & $0,76^{\star \star \star}$ & $0,76^{* * *}$ & $0,78^{\star * *}$ & $0,048^{\mathrm{NS}}$ & $0,81^{\star \star *}$ & $0,27^{*}$ & $0,41^{\star * *}$ & $0,29^{*}$ & $-0,01^{\mathrm{NS}}$ & $0,31^{*}$ & 0,22 & 0,21 . & $0,34^{* *}$ \\
\hline $\begin{array}{l}\text { Coxão } \\
\text { Mole }\end{array}$ & $0,79^{\star \star \star}$ & $0,82^{* \star *}$ & $0,87^{* \star *}$ & $0,075^{\mathrm{NS}}$ & $0,88^{\star \star *}$ & $0,36^{* *}$ & $0,38^{* *}$ & $0,29^{*}$ & $-0,04^{\mathrm{NS}}$ & $0,39^{* *}$ & $0,27^{*}$ & $0,09^{\mathrm{NS}}$ & $0,32^{* *}$ \\
\hline $\begin{array}{l}\text { Filé Mig- } \\
\text { non }\end{array}$ & $0,37^{* *}$ & $0,39^{* *}$ & $0,40^{\star * *}$ & $0,20^{\mathrm{NS}}$ & $0,42^{\star * *}$ & $0,14^{\mathrm{NS}}$ & $0,14^{\mathrm{NS}}$ & $0,18^{\mathrm{NS}}$ & $0,16^{\mathrm{NS}}$ & $0,15^{\mathrm{NS}}$ & $0,48^{* * *}$ & $-0,003^{\mathrm{NS}}$ & $-0,03^{\mathrm{NS}}$ \\
\hline Maminha & $0,46^{\star * *}$ & $0,63^{\star \star \star}$ & $0,58^{\star \star *}$ & $0,17^{\mathrm{NS}}$ & $0,57^{* * *}$ & $0,28^{*}$ & $0,17^{\mathrm{NS}}$ & 0,23 & 0,23 & 0,24 & $0,26^{*}$ & $0,17^{\mathrm{NS}}$ & $0,43^{\star \star \star}$ \\
\hline M.T & $0,72^{\star \star \star}$ & $0,68^{* * *}$ & $0,68^{* * *}$ & $0,057^{\mathrm{NS}}$ & $0,72^{\star \star \star}$ & $0,29^{*}$ & $0,38^{* *}$ & $0,25^{*}$ & $-0,08^{\mathrm{NS}}$ & $0,30^{*}$ & $0,26^{*}$ & $-0,02^{\mathrm{NS}}$ & $0,30^{*}$ \\
\hline Patinho & $0,72^{\star \star *}$ & $0,77^{* \star *}$ & $0,79^{* * *}$ & $0,029^{\mathrm{NS}}$ & $0,84^{\star \star *}$ & $0,24^{*}$ & $0,48^{* * *}$ & 0,21 . & $-0,08^{\mathrm{NS}}$ & $0,38^{\star *}$ & $0,29^{*}$ & $0,13^{\mathrm{NS}}$ & $0,29^{*}$ \\
\hline Picanha & $0,57^{\star \star *}$ & $0,60^{\star \star \star *}$ & $0,59^{\star \star *}$ & $0,17^{\mathrm{NS}}$ & $0,59^{\star \star \star}$ & $0,36^{* *}$ & $0,18^{\mathrm{NS}}$ & $0,34^{* *}$ & $0,24^{*}$ & 0,22 & 0,21 . & $0,11^{\mathrm{NS}}$ & $0,28^{*}$ \\
\hline T.E & $0,82^{* * *}$ & $0,88^{* * *}$ & $0,90^{\star \star *}$ & $0,11^{\mathrm{NS}}$ & $0,93^{* * *}$ & $0,37^{* *}$ & $0,41^{* * *}$ & $0,30^{*}$ & $0,06^{\mathrm{NS}}$ & $0,41^{* * *}$ & $0,36^{\star *}$ & $0,12^{\mathrm{NS}}$ & $0,35^{\star *}$ \\
\hline
\end{tabular}

$\mathrm{PC}=$ peso corporal; $\mathrm{PCQ}=$ peso da carcaça quente; $\mathrm{PCF}=$ peso da carcaça fria; $\mathrm{RCQ}=$ rendimento de carcaça quente; $\mathrm{PMC}=$ peso da meia carcaça; C. Int= comprimento interno; C.Per= comprimento da perna; L.Per= largura da perna; P.Per=Profundidade perna; P.Pei= profundidade peito; $\mathrm{PE} . P e r=$ perímetro perna; $\mathrm{AOL}=$ área do músculo Longissimus; $E G S=e s p e s s u r a$ de gordura subcutânea; $C D / L a g a r t o=$ cortes do coxão duro; M.T=músculo traseiro; T.E= cortes do traseiro especial $\left({ }^{* *} P<0.001,{ }^{* *} P<0.01,{ }^{*} P<0.05, \cdot P<0.1\right)$.

$69,89 \%$ (MC) para o patinho, 35, 12\% (PCQ) para a picanha (Tabela VI), dessa forma a inclusão da variável peso é fundamental para estimar a produção de cortes comerciais, isto é, o peso é o principal componente que deve ser levado em consideração para os cortes comerciais em fêmeas bovinas, pois o mesmo foi capaz de explicar cerca de $60 \%$ dos modelos de predição.

O acréscimo de contribuição das demais medidas realizadas na carcaça (comprimento, largura, profundidade, perímetro, espessura de gordura subcutânea e área de olho de lombo) para a predição dos cortes comerciais do traseiro especial foi baixo (Tabela VI), ressaltando que a formação dos cortes comerciais está relacionada intimamente com o peso desses animais, e as medidas morfométricas da carcaça sozinhas não contribuem de forma isolada para a formação dos cortes do traseiro especial de fêmeas bovinas.

A fim de estabelecer a validação das equações de regressão para os cortes comerciais do dianteiro e traseiro especial foram calculados e comparados os valores observados e preditos (Figura 1). Os valores observados e preditos foram semelhantes para todos os cortes do dianteiro e traseiro, com diferença mínima de 0,5 entre os valores, explicando assim a alta capacidade de predição das equações de regressão, ou seja, as medidas inclusas nas equações de regressão contribuem para formação dos melhores indivíduos de corte da categoria fêmea, confirmando que tais equações podem ser aplicadas para estimar a retalhabilidade dos cortes comerciais de novilhas nelore. 


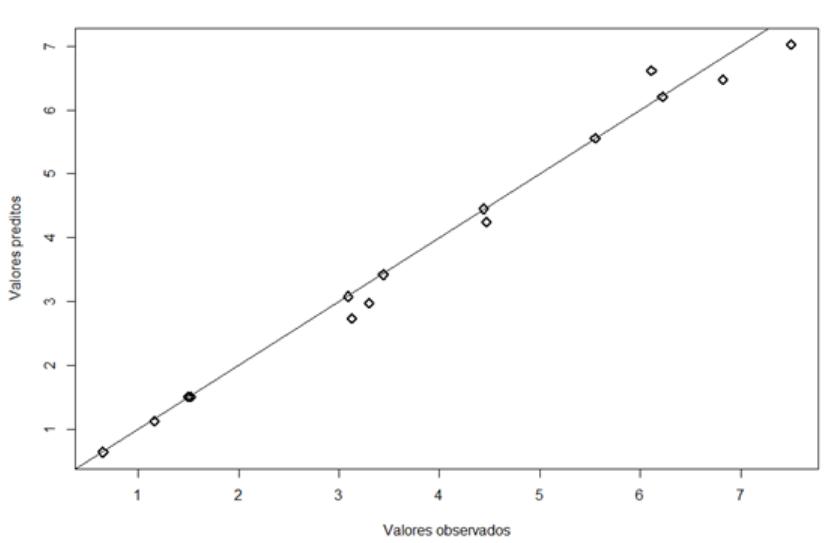

Figura 1. Validação das equações de regressão para os cortes comerciais do dianteiro e traseiro especial foram calculados e comparados os valores observados e preditos (Validation of regression equations for the commercial cuts of the special front and rear were calculated and compared the observed and predicted values).

Tabela IV. Modelo de predição (stepwise) para o peso dos cortes comerciais do dianteiro e traseiro especial de novilhas Nelore. (Prediction model (stepwise) for the weight of the commercial cuts of the front and the special rear of Nellore heifers).

\begin{tabular}{|c|c|c|c|c|c|}
\hline $\begin{array}{l}\text { Variáveis } \\
\text { Dependentes }\end{array}$ & \multicolumn{2}{|c|}{$\begin{array}{l}\text { Variáveis } \\
\text { Independentes }\end{array}$} & Intercepto & \multirow[t]{2}{*}{$\mathrm{R}^{2}$} & p-valor \\
\hline \multicolumn{5}{|c|}{ CORTES COMERCIAIS DO DIANTEIRO } & \\
\hline \multirow{3}{*}{ C.D } & PC & & 0,0146 & & \\
\hline & PMC & $-1,54$ & 0,1856 & \multirow[t]{2}{*}{$84,13 \%$} & \multirow[t]{2}{*}{$<0,001$} \\
\hline & AOL & & 0,0299 & & \\
\hline \multicolumn{6}{|c|}{ CORTES COMERCIAIS DO TRASEIRO ESPECIAL } \\
\hline \multirow{6}{*}{ T.E } & PC & & 0,0163 & \multirow{6}{*}{$88,66 \%$} & \multirow{6}{*}{$<0,001$} \\
\hline & PMC & & 0,2705 & & \\
\hline & AOL & -35 & 0,0426 & & \\
\hline & C.Per & $-6,35$ & 0,0755 & & \\
\hline & PE.Per & & 0,0367 & & \\
\hline & EGS & & $-0,6155$ & & \\
\hline
\end{tabular}

$\mathrm{R}^{2}=$ Coeficiente de determinação; $\mathrm{p}$-valor= valor de $\mathrm{p} ; \mathrm{C} . \mathrm{D}=$ cortes do dianteiro; $\mathrm{PC}=$ peso corporal; $\mathrm{PMC}=$ peso da meia carcaça; $\mathrm{AOL}=$ área do músculo Longissimus; $\mathrm{T}$.E=cortes do traseiro especial; C.Per= comprimento da perna; PE.Per= perímetro da perna; EGS= espessura de gordura subcutânea.

Na Tabela VII estão os dados numéricos referentes aos valores observados e preditos. O comportamento dos mesmos foi altamente semelhante, com ênfase na validação das equações realizadas pelo método stepwise para predizer os cortes comerciais com relação às medidas realizadas na carcaça, confirmando assim a semelhança dos valores observados no gráfico I. Concomitante com os valores altos a moderados do $\mathrm{R}^{2}$ de cada modelo de regressão dos cortes comerciais, uma vez que quanto mais alto o valor de $\mathrm{R}^{2}$ melhor o modelo ajusta seus dados.

\section{DISCUSSÃO}

A variável peso é altamente correlacionada com os cortes cárneos comerciais, sendo presente em vá- rios procedimentos de classificação de carcaça, por ser uma medida que influência diretamente na deposição de músculo e gordura de um animal. Vaz et al. (2012), ressaltaram correlação de 0,97 para o quarto dianteiro e o peso da carcaça quente de bovinos machos e fêmeas, o mesmo foi verificado no trabalho com correlação de 0,89 para essa variável.

Dessa forma Cyrillo et al. (2012), estudando medidas de carcaça de bovinos da raça Nelore x Guzerá e Caracu, encontrou correlação ligeiramente superior para peso vivo de 0,79 com o acém e 0,76 para paleta, essa superioridade pode ser explicada pelo fato de animais machos possuírem carcaças mais pesadas em relação as fêmeas, assim como animais machos apresentam maiores pesos e rendimento do dianteiro, consequentemente maiores pesos do acém, peixinho, paleta e músculo, devido a diferenciação sexual, proporcionada pela ação dos hormônios andrógenos que são associados à maior deposição muscular no organismo (Laurindo et al., 2015).

Para avaliação das características de carcaça as medidas morfométricas estão sendo cada vez mais exploradas e utilizadas para auxiliar na predição do melhor individuo. No presente trabalho as mesmas não apresentaram altas correlações, porém isso pode ter ocorrido devido ao sexo dos animais, uma vez que fêmeas não possuem um desenvolvimento muscular tão acentuado como animais machos, concomitante ao fato de serem lotes heterogêneos, uma vez que a homogeneidade do lote garante um melhor grau de acabamento (gordura subcutânea) e uniformidade das carcaças.

Estudos realizados por Silva et al. (2012) com bovinos Nelore mostram correlação de 0,69 entre a área de olho de lombo e o peso dos cortes do traseiro especial, o mesmo foi relatado por Maeno et al. (2014) que encontrou correlação de 0,69 para bovinos machos, superior ao encontrado no presente trabalho, indicando que o sexo dos animais possui importância significativa sobre essa variável, sendo assim, animais machos de grande porte voltado para a produção de carne aponta maior desenvolvimento muscular e consequentemente maior área do músculo Longissimus.

Cyrillo et al. (2012), estudaram medidas corporais de bovinos da raça Nelore e Guzerá (Zebu) e Caracu e encontraram correlação de 0,24 entre o comprimento do corpo e o corte contra-filé, também não encontraram correlação entre as medidas corporais e o corte filé mignon. Em estudos realizados por Costa et al. (2002) sobre características de carcaça com novilhos da raça Aberdeen Angus, verificara correlação de $0,77,0,62$ entre o comprimento da carcaça, comprimento da perna com o peso dos cortes do traseiro, respectivamente.

Segundo Tauroco et al. (2007), a variável peso é capaz de explicar a maior parte das diferenças dos valores dos coeficientes de determinação entre as equações a partir de medida realizadas na carcaça, obtidas para a predição da porção comestivel da carcaça. A variável fundamental para o ponto de vista comercial é o peso, uma vez que sua elevação 
Tabela V. Participação das variáveis obtidas na carcaça para a predição do peso dos cortes do dianteiro de novilhas Nelore (Participation of the variables obtained in the carcass for the prediction of the weight of the front cuts of Nellore heifers).

\begin{tabular}{|c|c|c|c|c|c|}
\hline Variáveis dependentes & Variáveis independentes & Intercepto & Coeficiente de Regressão & $\mathrm{R}^{2}$ & $p$-valor \\
\hline \multirow[t]{8}{*}{ Pescoço } & PCF & 3,41 & 0,0067 & 0,4026 & $<0,001$ \\
\hline & $\mathrm{RCQ}$ & & 0,0448 & 0,403 & $<0,001$ \\
\hline & $P C Q$ & & $-0,0375$ & 0,4207 & $<0,001$ \\
\hline & PE.Per & & $-0,0223$ & 0,4386 & $<0,001$ \\
\hline & $\mathrm{AOL}$ & & 0,0171 & 0,4436 & $<0,001$ \\
\hline & C.Int & & $-0,0328$ & 0,4523 & $<0,001$ \\
\hline & P.Pei & & 0,0261 & 0,4618 & $<0,001$ \\
\hline & L.Per & & $-0,0571$ & 0,476 & $<0,001$ \\
\hline \multirow[t]{3}{*}{ Acém } & PC & $-0,67$ & 0,0102 & 0,5403 & $<0,001$ \\
\hline & PMC & & 0,0357 & 0,5726 & $<0,001$ \\
\hline & $\mathrm{RCQ}$ & & $-0,0194$ & 0,5887 & $<0,001$ \\
\hline \multirow[t]{5}{*}{ Capa de Paleta } & P.Pei & $-0,12$ & 0,0112 & 0,0099 & 0,0056 \\
\hline & PE.Per & & $-0,0150$ & 0,1363 & 0,0037 \\
\hline & AOL & & 0,0059 & 0,1778 & 0,0016 \\
\hline & PCF & & 0,0073 & 0,1794 & 0,0028 \\
\hline & $P C Q$ & & $-0,0062$ & 0,1982 & 0,0024 \\
\hline \multirow[t]{5}{*}{ Miolo da Paleta } & PMC & $-1,28$ & 0,0387 & 0,6125 & $<0,001$ \\
\hline & PE.Per & & 0,0141 & 0,6764 & $<0,001$ \\
\hline & P.Pei & & $-0,0157$ & 0,687 & $<0,001$ \\
\hline & $\mathrm{RCQ}$ & & 0,0109 & 0,6942 & $<0,001$ \\
\hline & $\mathrm{PC}$ & & 0,0033 & 0,7002 & $<0,001$ \\
\hline \multirow[t]{5}{*}{ Músculo Dianteiro } & PMC & 0,49 & 0,0503 & 0,5009 & $<0,001$ \\
\hline & PCF & & $-0,0257$ & 0,5057 & $<0,001$ \\
\hline & $P C Q$ & & 0,0147 & 0,5346 & $<0,001$ \\
\hline & $\mathrm{RCQ}$ & & $-0,0133$ & 0,5441 & $<0,001$ \\
\hline & PE.Per & & 0,0084 & 0,5675 & $<0,001$ \\
\hline \multirow[t]{3}{*}{ Paleta } & PMC & 0,33 & 0,0305 & 0,5886 & $<0,001$ \\
\hline & P.Pei & & 0,0156 & 0,6155 & $<0,001$ \\
\hline & C.Per & & $-0,0155$ & 0,6262 & $<0,001$ \\
\hline
\end{tabular}

$\mathrm{R} 2=$ Coeficiente de determinação; $p$-valor= valor de $\mathrm{p} ; \mathrm{PC}=$ peso corporal; $\mathrm{PCF}=$ peso da carcaça fria; $\mathrm{RCQ}=\mathrm{rendimento}$ de carcaça quente; $\mathrm{PCQ}=$ peso da carcaça quente; $\mathrm{PE} . \mathrm{Per}=$ perímetro perna; $\mathrm{AOL=}$ área do músculo Longissimus; $\mathrm{C}$.Int= comprimento interno; $\mathrm{P} . \mathrm{Pei}=$ profundidade peito; L.Per= largura perna; $\mathrm{PMC}=$ peso da meia carcaça; C.Per= comprimento perna.

redunda influência na composição tecidual (Siqueira et al., 2001).

O aumento no peso corporal de abate resulta em um aumento no peso absoluto do dianteiro, ressaltando que a manutenção da proporção do dianteiro em relação ao aumento da variável peso indica que este corte cresce em uma determinada taxa semelhante à da carcaça como um todo, como também evidenciado no presente estudo (Missio et al., 2015).

Uma das principais características na carcaça bovina é causada pelo dimorfismo sexual, uma vez que machos inteiros apresentam maiores pesos finais de carcaça, o que caracteriza a maior taxa de ganho, especialmente direcionada para a porção dianteira do animal. Machos inteiros apresentam rendimento de acém mais elevados em relação aos machos castrados e fêmeas, em função do maior peso de abate de animais não-castrados (Marcondes et al., 2008). Luchiari et al. (2000), relata que o touro é o animal que utiliza todo potencial para desenvolver músculo diferencialmente, podendo explicar a baixa contribuição das demais medidas da carcaça como comprimento, largura, profundidade, perímetro e área de olho de lombo. 


\begin{tabular}{|c|c|c|c|c|c|}
\hline Variáveis dependentes & Variáveis independentes & Intercepto & Coeficiente de Regressão & $\mathrm{R}^{2}$ & $\mathrm{p}$-value \\
\hline \multirow[t]{12}{*}{ Alcatra } & $\mathrm{PCQ}$ & $-0,26$ & 0,0145 & 0,6381 & $<0,001$ \\
\hline & EGS & & $-0,1183$ & 0,6594 & $<0,001$ \\
\hline & $\mathrm{RCQ}$ & & 0,0145 & 0,6626 & $<0,001$ \\
\hline & $\mathrm{PC}$ & & 0,0041 & 0,6827 & $<0,001$ \\
\hline & P.Pei & & 0,0096 & 0,6924 & $<0,001$ \\
\hline & C.Per & & 0,0156 & 0,7028 & $<0,001$ \\
\hline & L.Per & & $-0,0231$ & 0,7001 & $<0,001$ \\
\hline & PE.Per & & $-0,0052$ & 0,7003 & $<0,001$ \\
\hline & C.Int & & $-0,0105$ & 0,7027 & $<0,001$ \\
\hline & $\mathrm{AOL}$ & & 0,0046 & 0,7031 & $<0,001$ \\
\hline & $P C Q$ & & $-0,0069$ & 0,7063 & $<0,001$ \\
\hline & P.Per & & 0,00984 & 0,7097 & $<0,001$ \\
\hline \multirow[t]{4}{*}{ Contra Filé } & PMC & $-1,16$ & 0,0446 & 0,5849 & $<0,001$ \\
\hline & PE.Per & & 0,0173 & 0,6246 & $<0,001$ \\
\hline & EGS & & $-0,1443$ & 0,6295 & $<0,001$ \\
\hline & $P C Q$ & & 0,0127 & 0,6353 & $<0,001$ \\
\hline \multirow[t]{4}{*}{ C.D/Lagarto } & PMC & 2,24 & 0,0392 & 0,6553 & $<0,001$ \\
\hline & $\mathrm{PC}$ & & 0,0061 & 0,6669 & $<0,001$ \\
\hline & $\mathrm{AOL}$ & & 0,0108 & 0,6766 & $<0,001$ \\
\hline & C.Int & & $-0,0212$ & 0,6896 & $<0,001$ \\
\hline \multirow[t]{5}{*}{ Coxão Mole } & PMC & 0,79 & 0,0647 & 0,7768 & $<0,001$ \\
\hline & EGS & & $-0,1728$ & 0,7926 & $<0,001$ \\
\hline & P.Per & & $-0,0289$ & 0,8007 & $<0,001$ \\
\hline & PC & & 0,0039 & 0,8049 & $<0,001$ \\
\hline & $\mathrm{AOL}$ & & 0,0084 & 0,8092 & $<0,001$ \\
\hline \multirow[t]{3}{*}{ Filé Mignon } & PE.Per & $-0,47$ & 0,0135 & 0,2139 & $<0,001$ \\
\hline & PMC & & 0,0117 & 0,2957 & $<0,001$ \\
\hline & $\mathrm{AOL}$ & & $-0,0078$ & 0,3319 & $<0,001$ \\
\hline \multirow[t]{3}{*}{ Maminha } & $P C Q$ & $-0,43$ & 0,0054 & 0,3909 & $<0,001$ \\
\hline & $\mathrm{AOL}$ & & 0,0050 & 0,4334 & $<0,001$ \\
\hline & P.Per & & 0,0079 & 0,4443 & $<0,001$ \\
\hline \multirow[t]{5}{*}{ M.T } & PC & 1,14 & 0,0043 & 0,5106 & $<0,001$ \\
\hline & EGS & & $-0,1183$ & 0,5462 & $<0,001$ \\
\hline & PMC & & 0,0132 & 0,5933 & $<0,001$ \\
\hline & P.Per & & $-0,0175$ & 0,6154 & $<0,001$ \\
\hline & $\mathrm{AOL}$ & & 0,0049 & 0,6237 & $<0,001$ \\
\hline \multirow[t]{5}{*}{ Patinho } & PMC & $-0,69$ & 0,0395 & 0,6989 & $<0,001$ \\
\hline & C.Per & & 0,0205 & 0,7097 & $<0,001$ \\
\hline & EGS & & $-0,0825$ & 0,7189 & $<0,001$ \\
\hline & $\mathrm{AOL}$ & & 0,0066 & 0,7257 & $<0,001$ \\
\hline & P.Per & & $-0,0152$ & 0,7325 & $<0,001$ \\
\hline \multirow[t]{2}{*}{ Picanha } & $P C Q$ & $-0,75$ & 0,0078 & 0,3512 & $<0,001$ \\
\hline & L.Per & & 0,0270 & 0,3976 & $<0,001$ \\
\hline
\end{tabular}

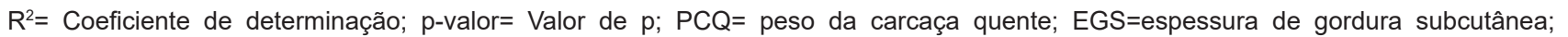
$\mathrm{RCQ}=$ rendimento de carcaça quente; $\mathrm{PC}=$ peso corporal; $\mathrm{P} . \mathrm{Pe} \mathrm{i}=$ profundidade peito; $\mathrm{C} . P e r=$ comprimento perna; L.Per=largura perna; $\mathrm{PE} . P e r=$ perímetro perna; $C . I n t=$ comprimento interno; $A O L=a ́ r e a$ do músculo Longissimus; $P . P e r=$ profundidade perna; $P M C=p e s o$ da meia carcaça; CD/Lagarto= corte do coxão duro. 


\begin{tabular}{ccc}
\hline Tabela VII. Valores observados e preditos dos cortes comerciais do dianteiro e traseiro especial de novilhas \\
Nelore (Observed and predicted values of commercial front and rear cuts of Nellore heifers). \\
\hline Variáveis dependentes & Valor Observado & Valor Predito \\
Pescoço & 6,22 & 6,21 \\
Acém & 5,55 & 5,56 \\
Capa de Paleta & 0,65 & 0,64 \\
Miolo da Paleta & 4,44 & 4,45 \\
Paleta & 3,09 & 3,08 \\
Músculo Dianteiro & 3,44 & 3,42 \\
Coxão Duro/Lagarto & 6,11 & 6,62 \\
Coxão Mole & 7,50 & 7,02 \\
Patinho & 4,47 & 4,24 \\
Alcatra & 3,13 & 2,74 \\
Contra Filé & 6,82 & 6,47 \\
Filé Mignon & 1,50 & 1,51 \\
Picanha & 1,52 & 1,51 \\
Maminha & 1,16 & 1,13 \\
Músculo Traseiro & 3,30 & 2,98 \\
\hline
\end{tabular}

Visto que como exposto por Bueno et al. (2000), o comprimento interno carcaça pode ser considerado um bom indicador de características de carcaça, devido alta relação com a porcentagem de ossos, peso de músculo e gordura da carcaça. Oliver et al. (2010), também relata que a largura e a convexidade da carcaça podem contribuir para a precisão da estimativa do rendimento de carne e cortes.

Silva et al. (2012) aplicando equações de regressão para prever a participação de características de carcaça do traseiro especial, relatou que $97 \%$ da variação do peso foi representada pelas características de carcaça, onde estava incluído na predição o peso da carcaça quente. Assim como no estudo de Jardim et al. (1985) afirmam que incluindo o comprimento da carcaça nas equações de regressão, o acréscimo foi baixo para predizer do peso do traseiro especial. Esse baixo acréscimo pode ser explicado pela diferença entre os sexos para as medidas morfométricas da carcaça, demonstrado por Siqueira et al. (2001) que o comprimento da perna, assim como o comprimento interno e externo da carcaça é maior em animais machos, devido ao maior alongamento ósseo em relação ás fêmeas, pois a fisiologia do macho apresenta taxa de crescimento mais elevada.

A área de olho de lombo é altamente correlacionada em vários estudos com os cortes comerciais do traseiro especial, como em um trabalho realizado por Tauroco et al. (2007), onde a inclusão da AOL nas equações de predição elevou a explicação para $63 \%$, esclarecendo que é a variável mais intensamente correlacionada com a porcentagem do traseiro especial. $\mathrm{O}$ mesmo não foi encontrado no presente trabalho visto que a área do músculo Longissimus é maior em machos do que fêmeas, pois é uma medida relacionada com a musculosidade da carcaça e con- sequentemente com a conformação que em geral é melhor em machos.

No presente estudo utilizando equações de regressão pelo método stepwise o resultado foi satisfatório, do mesmo modo foi afirmado por Lee et al. (2016) que utilizando equações de regressão pelo método de stepwise permitiu estimar bem a porcentagem do peso de cortes comerciais de bovinos, sendo que o valor previsto e o observado foram semelhantes para essa variável, concluindo que o peso da carcaça foi a principal variável para estimar o peso dos cortes comerciais, peso da gordura subcutânea e dos ossos.

A utilização de equações de regressão pelo método stepwise pode ser aplicado com maior eficácia para estimar cortes comerciais em novilhas Nelore quando comparado a outras equações como de Hankins \& Howe (1946), confirmado por Souza et al. (2019) onde essa equação não foi capaz de estimar o conteúdo de tecido adiposo da carcaça apresentando baixa precisão. $\mathrm{O}$ mesmo explica que existe a probabilidade que as equações desenvolvidas antes dos anos 80 podem ter uma aplicação limitada para pecuária moderna, devido a importantes mudanças no potencial de crescimento desses animais, como seu peso ao nascer, desmame e abate.

Da mesma maneira, Marcondes et al. (2009) relatam que sob diferenças sexuais, como machos inteiros, machos castrados e fêmeas as mesmas equações não foram uma boa estimativa para o tecido na carcaça de Nelore, em contrapartida no presente estudo podemos observar que o modelo utilizado para estabelecer as equações de regressão foi uma boa estimativa para a classe sexual fêmeas, podendo ser 
altamente aplicado para estimar os cortes comerciais do dianteiro e traseiro especial.

\section{CONCLUSÃO}

As medidas morfométricas realizadas na carcaça podem estimar as relações com o peso dos cortes comerciais em novilhas nelores. As melhores correlações com o peso dos cortes comerciais foram relacionas com o peso da carcaça. O peso da meia carcaça foi à medida mais importante para a predição dos cortes comerciais do dianteiro e traseiro especial.

\section{BIBLIOGRAFÍA}

Brasil - Ministério da Agricultura. R.I.I.S.P.O.A. 1980. Regulamento da Inspeção Industrial e Sanitária de Produtos de Origem Animal (Aprovado pelo decreto $n^{\circ} 30690$, de 20.03.52, alterado pelo decreto no 1255, de 25.06.52). Brasília, pp.66.

Bueno, MS, Cunha, EA, Santos, LE, Roda, DS, Leinz, FF 2000, 'Características de Carcaça de Cordeiros Suffolk Abatidos em Diferentes Idades', Revista Brasileira de Zootecnia, vol.29, no.6, pp. 1803-1810.

Costa, ECC, Vaz, FN, Filho, DCA, Bernardes, RALC, Kuss, F 2002, 'Características da Carcaça de Novilhos Red Angus Superprecoces Abatidos com Diferentes Pesos', Revista Brasileira de Zootecnia, vol.31, no. 1, pp.119-128.

Cyrillo, JNSG, Nardon, RF, Mercadante, MEZ, Bonilha, SFM, Arnandes, RHB 2012, 'Relações entre medidas biométricas, características de carcaça e cortes cárneos comerciais em bovinos zebu e caracu', Boletim da Indústria Animal, vol.69, no.1, pp.071-077. Jardim, POC, Ziegler, JCS, Osório, JCS 1985, 'Predição dos principais cortes da carcaça em novilhos', Pesquisa Agropecuária Brasileira, vol.20, no.2, pp.253-258.

Lage, JF, Paulino, SC, Valadares Filho, SC, Duarte, MS, Benedeti, PDB, Souza, NKP, COX, RB 2012, 'Influence of genetic type and level of concentrate in the finishing diet on carcass and meat quality traits in beef heifers', Meat Science, vol.90, no.3, pp. 770-77.

Lee, J, WON, S, Lee, J, Kim, J 2016, 'Prediction of Carcass Composition Using Carcass Grading Traits in Hanwoo Steers', Journal of Animal Science, vol. 29, no.9, pp. 1215-1221.

Luchiari Filho, A 2000, 'A pecuária da carne bovina', São Paulo, pp. 124.

Maeno, H, Oishi, K, Mitsuhashi, T, Kumagai, H, Hirooka, H 2014, 'Prediction of carcass composition and individual carcass cuts of Japanese Black steers' Meat Science, vol.98, no.3, pp.1365-1370.

Marcondes, MI, Filho, SCV, Paulino, PVR, Detmann, E, Valadares, RF, Santos, TR, Diniz, LL 2009, 'Predição da composição corporal e da carcaça a partir da seção entre a 9a e 11 a costelas em bovinos Nelore', Revista Brasileira de Zootecnia, vol.38, no.8, pp.1597-1604

Missio, RL, Restle, J, Moletta, JL, Kuss, F, Neiva, JNM, Elejalde, DAG, Moura, ICF, Prado, IN, Miotto, FRC 2015, 'Slaughter weights on animal performance, carcass commercial cuts and meat characteristics of cull cows. Semina: Ciências Agrárias, vol.36, no.6, pp.3827-3842.

Oliver, A, Mendizabal, JA, Ripoll, G, Albertí, P, Purroy, A 2010, 'Predicting meat yields and commercial meat cuts from carcasses of young bulls of Spanish breeds by the SEUROP method and an image analysis system', Meat Science, vol.84, pp. 628-633.
Paulino, PV, Filho, SCV, DETMANN, E, VALADARES, RFD, FONSECA, MA, VÉRAS, RM, OLIVEIRA, DM 2008, 'Desempenho produtivo de bovinos Nelore de diferentes classes sexuais alimentados com dietas contendo dois níveis de oferta de concentrado' Revista Brasileira de Zootecnia, vol.37, no.6, pp.1079-1087.

Pinheiro, RSB, Jorge, AM, Yokoo, MJ 2010, 'Correlações entre medidas determinadas in vivo por ultrassom e na carcaça de oveIhas de descarte' Revista Brasileira de Zootecnia, vol.39, no.5, pp.1161-1167.

Rezende, PLP, Restle, J, Padua, TJ, FernandeS, RJJ, LINO, AF, GUIMARÃES, PT 2013, 'Carcass morphometry of crossbred steers subjected to different nutritional strategies in the growing and finishing phases' Acta Scientiarum, vol.35, no.2.

Ribeiro, FG, Jorge, AM, Francisco, CL, Castilhos, AM, Pariz, CM, Silva, MB 2015, 'Simbióticos e monensina sódica no desempenho e na qualidade da carne de novilhas mestiças Angus confinadas' Pesquisa Agropecuária Brasileira, vol.50, no.10, pp.958-966.

Silva, SL. Tarouco, JU, Ferraz, JBS, Gomes, RC, Leme, PR, Navajas, EA 2012, 'Prediction of retail beef yield, trim fat and proportion of high-valued cuts in Nellore cattle using ultrasound live measurements' Revista Brasileira de Zootecnia, vol.41, no.9, pp.2025-2031.

Siqueira, R, Simões, CD, Fernandes, S 2001, 'Efeito do Sexo e do Peso ao Abate sobre a Produção de Carne de Cordeiro. I. Velocidade de Crescimento, Caracteres Quantitativos da Carcaça, pH da Carne e Resultado Econômico' Revista Brasileira Zootecnia, vol. 30, no.3, pp. 844-848.

Souza, EJO, Filho, SCV, Torres, TR, Silva, DC, Silva, JRC 2019, 'Predicting body and carcase composition in Nellore heifers and their cross-breeds' Revista Ciência Agronômica, vol.50, no. 1, pp. 148-158.

Suguisawa, L, Mattos, WRS, Oliveira, HN, Silveira, AC, Arrigoni, MB, Souza, AA 2006, 'Correlações simples entre as medidas de ultra-som e a composição da carcaça de bovinos jovens' Revista Brasileira de Zootecnia, vol.35, no. 1, pp.169-176.

Tarouco, JU, Lobato, JFP, Tarouco, AK, Massia, GIS 2007, 'Comparação entre medidas ultra-sônicas e da carcaça na predição da composição corporal em bovinos. Estimativas do peso e da porcentagem dos cortes comerciais do traseiro' Revista Brasileira de Zootecnia, vol.36, no.6, pp.2092-2101.

Vaz FN, Vaz RZ, Pascoal LL, Restle J, Leal WS, Ávila, MM 2012, 'Economical Analysis and Commercial Cuts Yields of Buffaloes and Bovines of Both Sexes' Journal of Animal Production Advances, vol. 10, no.2, pp.436-444

Vaz, FN, Vaz, RZ, Pascoal, LL, Pacheco, PS, Miotto, FRC, Teixiera, NP 2012, 'Análise econômica, rendimentos de carcaça e dos cortes comerciais de vacas de descarte $5 / 8$ hereford $3 / 8$ nelore abatidas em diferentes graus de acabamento' Ciência Animal Brasileira, vol.13, no.3, pp. 338-345. 